\title{
Wavelet Based Medical Image Watermarking Scheme for Patient Information Authenticity
}

\author{
Gokcen Cetinel ${ }^{*}{ }^{1}$, LLukman Cerkezi ${ }^{2}$
}

\section{Accepted $3^{\text {rd }}$ September 2016}

Abstract: Telemedicine is an important technique that permits transmission of medical and imaging data from one place to other, ensures the reliability of data and provides a convenient communications between patient and medical staff. In this study, Discrete Wavelet Transform (DWT) and Singular Value Decomposition (SVD) based medical image watermarking method that hides the patient information into medical image without affecting the image quality is proposed for the purpose of authentication. At the watermark (secret image containing patient information) embedding process, a chaotic map called as Arnold Cat Map (ACM) is applied to the watermark to improve the security of the method. So that the aim of the proposed watermarking scheme is personal authentication by ensuring the perceptual invisibility, PSNR (Peak Signal-to-Noise Ratio) values are calculated during the simulations. According to the experimental results, we can say that the proposed method provides higher PSNR values than compared current methods. Furthermore, in simulations DWT and SVD based watermarking scheme is implemented as chaotic and non-chaotic watermarking scheme to investigate the effect of chaotic map on the performance of the proposed algorithm.

Keywords: Medical image watermarking, discrete wavelet transform, singular value decomposition, PSNR.

\section{Introduction}

Telemedicine is a technique which aims to provide clinical and health assistance to remote areas by utilizing telecommunication and information technologies. Through using telemedicine, patients benefit from far away specialists for medical diagnosis without travelling. By telemedicine, it is possible to transmit medical and imaging data from one place to other and to communicate the patient with the medical staff. Transmitted medical images help physician to decide diagnostic procedures. Then, to evaluate the progress of treatment, telemedicine can be used again [1].

During the transmission, medical image may be destroyed by intruders or attacked by noise. We need to ensure reliable data transmission to not cause the wrong diagnosis. For this purpose, digital image watermarking is found to be an effective and promising mechanism by the researchers $[8,9]$.

Digital image watermarking is a technique of embedding a secret information (watermark) to a cover image. There are three common requirements for watermarking schemes: robustness, capacity and invisibility. However, there is a trade-off between these requirements. For example, a stronger watermark can be used to increase the robustness, but in this case watermark becomes noticeable. On the contrary, increasing the capacity decreases the robustness. Consequently, one must make a choice between these three requirements according to the application. For telemedicine technique, during the storing and transmission of medical images (i.e. Computed Tomography (CT), Magnetic Resonance Imaging (MRI) and X-ray imaging) the security of the digital data has a critical importance. Furthermore, we need to embed patient's information to the medical image without causing any perceptual changes. So, the perceptual invisibility of the watermarking scheme must be very high to maintain the quality of original medical image $[2,3]$.

\footnotetext{
${ }^{l}$ Electrical aand Electronics Engineering Department, Engineering Faculty, Sakarya University, Esentepe , 42031, Sakarya/Turkey

* Corresponding Author: Email: gcetinel@sakarya.edu.tr

Note: This paper has been presented at the $3^{\text {rd }}$ International Conference on Advanced Technology \& Sciences (ICAT'16) held in Konya (Turkey), September 01-03, 2016.
}

According to the embedding domain, watermarking techniques can be classified in to two groups as spatial domain watermarking techniques and frequency domain watermarking techniques. In spatial domain techniques, the secret data is directly inserted into the samples of the cover signal. In frequency domain techniques, embedding process is performed in the frequency domain by modifying the coefficients of the transformed image. The transformed image can be obtained by applying Discrete Cosine Transform (DCT), Discrete Fourier Transform (DFT), Discrete Wavelet Transform (DWT) or Redundant Discrete Wavelet Transform (RDWT) [4]. Although spatial domain techniques has low computational cost and are less complex, the transform domain techniques are most popular because of their significant advantages such as robustness and imperceptibility.

In this study, DWT and SVD based medical image watermarking scheme is proposed. In the proposed scheme, patient's information is embedded to the medical image as a binary watermark. The aim of this study is to embed the patient's information to the medical image without degrading the quality of medical image for personal authentication. At the watermark embedding stage, original cover (medical) image is decomposed by DWT into LL, LH, HL and HH sub-bands. Then SVD is applied to each sub-band. On the other side, ACM and SVD are performed for the original watermark, respectively. Then, the singular values of the medical image is added to the singular values of chaotic watermark in all sub-bands. Finally, by using invers SVD and inverse DWT the watermarked image is obtained.

The paper is organized as follows. In Section 2, in order to better understand the proposed algorithm, DWT, SVD and ACM are discussed. Watermark embedding and extracting algorithms are explained step by step in Section 3. Section 4 gives the experimental results and compares the proposed method with related studies. Finally, Section 5 concludes the paper.

\section{Applied Techniques}

In this section, the techniques used in the proposed method are explained briefly to better understand the watermark embedding and extraction steps.

\subsection{Discrete Wavelet Transform (DWT)}


DWT is a common tool used in watermarking schemes which transforms the image from spatial domain to frequency domain. It is shown in many papers that DWT is superior then other transform techniques in watermarking algorithms. By applying DWT, 1-D original signal is separated into the low-frequency and high frequency sub-bands. This process is called as decomposition and gives us DWT coefficients. The original signal can be reconstructed by using DFT coefficients. This reconstruction process is referred as inverse DWT (IDWT) [5].

By performing DWT on image (2-D signal) one time, the image is split into four sub-bands, $\mathrm{LL}_{1}, \mathrm{LH}_{1}, \mathrm{HL}_{1}$, and $\mathrm{HH} 1 . \mathrm{LL}_{1}$ is the low frequency sub-band and it contains the maximum of energy while the other sub-bands are middle $\left(\mathrm{LH}_{1}, \mathrm{HL}_{1}\right)$ and high $\left(\mathrm{HH}_{1}\right)$ frequency sub-bands. These middle and high frequency subbands represent the edges, outline, texture and other detail information of the original signal. If we want to perform again DWT to signal, we should apply the DWT to the $\mathrm{LL}_{1}$ sub-band. This process can be repeated until we get the appropriate decomposition level for our application.

Watermark embedding process is done by changing the wavelet coefficients according to the embedding algorithm. The watermark can be embedded either in low- frequency sub-band or high- frequency sub-band with respect to the purpose of the application. Embedding watermark to the high- frequency subband provides high imperceptibility advantage but robustness and stability of the scheme will be decreased. Robustness can be improved by embedding watermark to the middle a frequency sub-band but this will cause decrement of imperceptibility.

\subsection{Singular Value Decomposition (SVD)}

In recent years, SVD has become an extremely valuable tool in the analysis and the solution of problems especially in signal processing area. Image compression, data hiding, noise reduction and image watermarking are just some applications of SVD [6].

Given the data matrix A of dimension $\mathrm{N} x \mathrm{~N}$ which has the independent column (i.e. rank $(\mathrm{A})=\mathrm{N}$ ), there are two unitary matrices $\mathrm{V}$ and $\mathrm{U}$ such that

\section{$\mathbf{A}=\mathbf{U S V}^{\mathbf{T}}$}

(1)

where $\mathbf{S}=\operatorname{diag}\left(\sigma_{1}, \sigma_{2}, \ldots, \sigma_{\mathrm{W}}\right)$ is an diagonal matrix. Singular values are ordered as $\sigma_{1} \geq \sigma_{2} \geq \ldots \geq \sigma_{W}>0$. The Equation (1) is mathematical the representation of SVD.

SVD is an optimal decomposition method that concentrates the maximum signal energy into as few coefficients as possible. From the image processing point of view SVD has three main advantages: i) it can applied to any dimension of image matrix, ii) after applying SVD to image the singular values $S$ corresponds to the brightness of image while $\mathrm{U}$ and $\mathrm{V}$ denotes the geometric properties of the image, iii) the slight variations of singular values of an image may not affect the human visual perception. This stability property of SVD is the main reason why it is preferred for watermarking applications [7].

\subsection{Arnold's Cat Map (ACM)}

Chaotic signals has very broad range of application in secure communications, signal processing and cryptography because of their inherent properties that can be taken into account such as high complexity. There are many chaotic maps in literature appropriate for image processing applications. ACM is one of the most famous chaotic map used for randomizing the pixel locations in the image matrix. This randomizing provides high security for the image watermarking schemes. ACM can be applied for only square matrix. For $\mathrm{N} x \mathrm{~N}$ square image matrix it can be expressed as $\left[\begin{array}{l}x_{n+1} \\ y_{n+1}\end{array}\right]=\left[\begin{array}{cc}1 & a \\ b & a b+1\end{array}\right]\left[\begin{array}{l}x_{n} \\ y_{n}\end{array}\right] \bmod N=\boldsymbol{A}\left[\begin{array}{l}x_{n} \\ y_{n}\end{array}\right] \bmod N$.

(2)

where $\left(x_{n}, y_{\mathrm{n}}\right)$ and $\left(x_{\mathrm{n}+1}, y_{\mathrm{n}+1}\right)$ are the locations of pixels before and after iterations, respectively. In Equation (2) the coefficients $a$ and $b$ are selected such as $\operatorname{det}(\mathbf{A})=1[5]$.

After applying a few iterations into original image the locations of pixel will be scrambled but when the iterations are repeated enough we will attain the original image again. There is no any formula for exact number of iterations for given dimension of image, but for higher dimension we expect higher iterations. ACM transformation for different iterations is illustrated in Fig.1. As a conclusion the number of iterations both in watermarking embedding and extracting must be selected carefully [10].

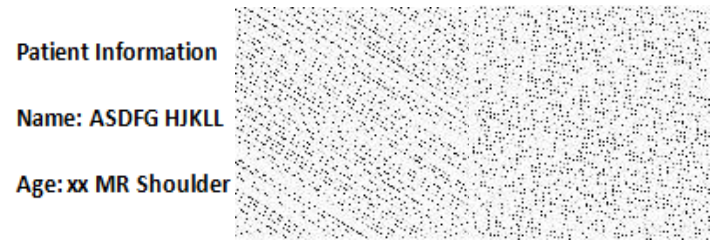

(a)

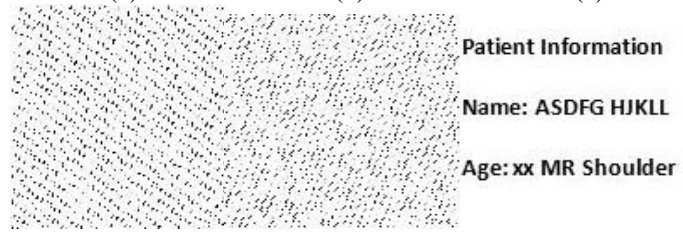

(d)

(e)

(f)
Figure 1. (a) Original watermark, (b) 3, (c) 37, (d) 76, (e) 87 and (f) 96th iteration ACM applied watermark image.

\section{Proposed Medical Image Watermarking Algorithm}

In this section, the proposed medical image watermarking algorithm including watermark embedding and watermark extraction are explained in detail with all steps.

\subsection{Watermark Embedding}

In our algorithm, CT, MRI and X-ray images are used as a cover image. During the embedding algorithm, two, three and four level decomposition of DWT are performed to these images, respectively. The steps of the embedding algorithm are given below:

1. By performing the appropriate decomposition level to cover image, the image is divided into LL, LH, HL and HH sub-bands.

2. SVD is applied to the each sub-band of the cover image as follows:

$$
\boldsymbol{A}^{i}=\boldsymbol{U}^{i} \boldsymbol{S}^{i} \boldsymbol{V}^{i}
$$

where $\boldsymbol{A}$ is the sub-band of cover image and $i$ denotes the LL, LH, HL and HH sub-bands, respectively.

3. We attain chaotic watermark $\boldsymbol{W}_{\mathrm{ACM}}$ by performing 76 iterations ACM to the watermark image $(\boldsymbol{W})$.

4. SVD is applied to chaotic watermark $\boldsymbol{W}_{\mathrm{ACM}}$ as follows:

$$
\boldsymbol{W}_{A C M}=\boldsymbol{U}_{A C M} \boldsymbol{S}_{A C M} V_{A C M}^{T}
$$


5. Singular values $\boldsymbol{S}^{i}$ of cover image are modified with singular values $\boldsymbol{S}_{A C M}$ of chaotic watermark image

$\boldsymbol{S}^{* i}=\boldsymbol{S}^{i}+\alpha \boldsymbol{S}_{A C M}$

where $\alpha$ is fixed scaling factor for all sub-bands and $i$ represents LL, LH, HL and HH sub-bands.

6. Inverse SVD is performed by using modified singular values

$\boldsymbol{A}^{* i}=\boldsymbol{U}^{i} \boldsymbol{S}^{* i} \boldsymbol{V}^{i^{T}}$

where $i$ represents LL, LH, HL and HH sub-bands, again.

7. Finally, we implement inverse DWT to the modified coefficients of related sub-bands in order to get the watermarked image.

\subsection{Watermark Extraction}

The steps of the watermark extraction algorithm are given below:

1. We decide the most appropriate level of DWT and applied DWT to the watermarked medical images. By this way, we get $\mathrm{LL}^{*}, \mathrm{LH}^{*}, \mathrm{HL}^{*}$ and $\mathrm{HH}^{*}$ sub-bands.

2. SVD is applied to $\mathrm{LL}^{*}, \mathrm{LH}^{*}, \mathrm{HL}^{*}$ and $\mathrm{HH}^{*}$ sub-bands, as follows:

$$
\boldsymbol{A}^{* i}=\boldsymbol{U}^{* i} \boldsymbol{S}^{* i} \boldsymbol{V}^{* i}
$$

where $I$ denotes LL, LH, HL and $\mathrm{HH}$ sub-bands respectively.

3. Singular values of watermark image are computed as follows:

$\boldsymbol{S}^{W}=\left(\boldsymbol{S}^{* i}-\boldsymbol{S}^{i}\right) / \alpha$

where $\alpha$ is fixed scale factor for all sub-bands.

4. By applying inverse SVD, we obtain the chaotic watermark

$$
\boldsymbol{W}_{A C M}^{*}=\boldsymbol{U}^{i} \boldsymbol{S}^{w} \boldsymbol{V}^{i^{T}}
$$

5. Finally, we perform 20 iterations $\mathrm{ACM}$ to $\boldsymbol{W}_{A C M}^{*}$, (for $128 \times 128$ image matrix96-76=20), in order to get the original watermark image.

\section{Experimental Results}

The purpose of this section is to investigate the efficiency of the proposed medical image watermarking method by performing simulations. In order to expand the application areas of the proposed scheme the CT, MRI and X-ray medical images are utilized. MATLAB computer program is used during the simulations. The size of CT image is $1024 \times 1024$, MRI image $512 \times 512$ and X-ray image is $2048 \times 2495$.The watermark image of size $128 \times 128$, includes the patient's information such as the name, surname, age and type of medical image. Since the medical images are used for diagnosis and treatment, the quality of medical image is crucial. So the imperceptibility of watermark must be very high. In order to investigate the imperceptibility of the watermarking schemes, the peak signal-to-noise ratio (PSNR) criterion is commonly used in the literature. PSNR can be defined as the similarity between the original cover (medical) image and watermarked medical image and can be calculated as follows,

$P S N R=10 \log _{10}\left[\frac{\max \left((X(i, j))^{2}\right.}{M S E}\right]$

where the mean square error (MSE) is defined as :

$M S E=\frac{1}{m x n} \sum_{i=1}^{m} \sum_{j=1}^{n}[\boldsymbol{X}(i, j)-\boldsymbol{Y}(i, j)]^{2}$

In Equation (11), size of the images are $m \times n$. The higher PSNR values means that the watermark is better hidden. The other criterion for evaluating the performance of algorithm is Normalized Correlation (NC) which measures the similarity between the original watermark and extracted watermark [2, 4]. It is computed from:

$N C=\frac{\sum_{k=1}^{m} \sum_{j=1}^{n}\left[\boldsymbol{W}(k, j) \boldsymbol{W}^{\prime}(k, j)\right]}{\sqrt{\sum_{k=1}^{m} \sum_{j=1}^{n}[\boldsymbol{W}(k, j)]^{2}} \sqrt{\sum_{k=1}^{m} \sum_{j=1}^{n}\left[\boldsymbol{W}^{\prime}(k, j)\right]^{2}}}$

where $\boldsymbol{W}$ and $\boldsymbol{W}^{\prime}$ represent the original and extracted watermarks, respectively. The correlation coefficient takes on values in the interval $[-1,1]$. If it is near 0 , the extracted watermark is completely uncorrelated with the original one. Generally, the NC is considered acceptable if it is 0.75 or above.

Table 1.Results for proposed medical image watermarking scheme.

\begin{tabular}{|l|c|c|c|c|}
\hline & $\begin{array}{c}\text { PSNR(dB) } \\
\text { (non-chaotic) }\end{array}$ & $\begin{array}{c}\text { NC (non- } \\
\text { chaotic) }\end{array}$ & $\begin{array}{c}\text { PSNR(dB) } \\
\text { (chaotic) }\end{array}$ & $\begin{array}{c}\text { NC } \\
\text { (chaotic) }\end{array}$ \\
\hline CT & & & & \\
\hline LL & 102.181 & 0.9945 & 90.4105 & 0.9977 \\
\hline LH & 98.2397 & 0.9983 & 87.0457 & 0.9955 \\
\hline HL & 97.9923 & 0.9979 & 86.9351 & 0.9966 \\
\hline HH & 69.8840 & 0.9961 & 86.9940 & 0.9982 \\
\hline MRI & & & & \\
\hline LL & 94.3808 & 0.9960 & 94.0275 & 0.9924 \\
\hline LH & 100.410 & 0.9942 & 91.5416 & 0.9942 \\
\hline HL & 100.432 & 0.9926 & 91.6404 & 0.9943 \\
\hline HH & 100.354 & 0.9856 & 91.4640 & 0.9970 \\
\hline X-ray & & & & \\
\hline LL & 92.3033 & 0.9928 & 93.6579 & 0.9953 \\
\hline LH & 96.7856 & 0.9936 & 94.7722 & 0.9960 \\
\hline HL & 96.8000 & 0.9870 & 94.8258 & 0.9951 \\
\hline HH & 93.3746 & 0.9976 & 94.8079 & 0.9964 \\
\hline
\end{tabular}

Table 2. Comparison between proposed scheme and reference (for subband LL) [2].

\begin{tabular}{|l|c|c|}
\hline & Method [2] & Proposed Method \\
\hline CT & $46.01 \mathrm{~dB}$ & $102.18 \mathrm{~dB}$ \\
\hline MRI & $47.20 \mathrm{~dB}$ & $94.38 \mathrm{~dB}$ \\
\hline x-Ray & $49.58 \mathrm{~dB}$ & $92.30 \mathrm{~dB}$ \\
\hline
\end{tabular}

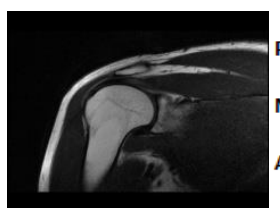

(a)
Patient Information Name: ASDFG HJKLL Age: $x x$ MR Shoulder (b)

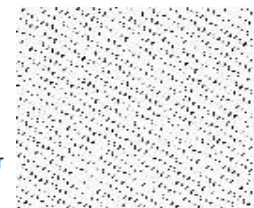

(c) 


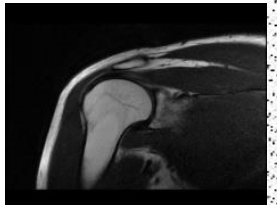

(d)

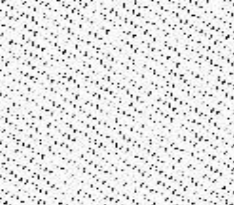

(e)
Patient Information

Name: ASDFG HJKLL

Age: xx MR Shoulder

(f)
Figure 2. (a)MR medical image, (b) original watermark, (c) chaotic watermark, (d) watermarked image, (e) extracted chaotic watermark, (f) extracted original watermark.

In Table 1, PSNR and NC values are given for CT, MR and Xray image. The watermarking algorithm is performed with and without chaos. As can be seen from the table, for both cases the PSNR values are almost over $90 \mathrm{~dB}$ and $\mathrm{NC}$ values are over 0.98 . According to these results it is clear that the imperceptibility of watermark in the proposed scheme ensures the desired demand. In the Table 2, the comparison between the proposed scheme and reference [2] is illustrated. Watermark is embedded in LL subband for comparison. The superiority of our method can be seen obviously. Furthermore, in Figure 2 each step of proposed watermarking scheme is shown with corresponding images. The other advantage of proposed scheme is that acquired imperceptibility values are independent from decomposition level of DWT.

\section{Conclusion}

In this study, DWT and SVD based medical image watermarking scheme is proposed. In the proposed scheme, patient's information is embedded to the medical image as a binary watermark. CT, MR and X-Ray images are used as medical image in the simulations. At the watermark embedding process, a chaotic map called as ACM is applied to the watermark to improve the security of the method.

The aim of the study is to hide patient's information to the medical image without degrading the quality of medical image for personal authentication. By calculating PSNR and NC values we prove that the proposed method ensures the desired property. Furthermore, we exhibit that the proposed method provides higher PSNR values than compared current methods. The proposed scheme can be applied independently of type and size of medical image.
[1] Eswaraiah, R., Reddy, E.S.,"Robust Medical Image Watermarking Technique for Accurate Detection of Tampers Inside Region of Interest and Recovering Origina Region of Interest”, IET Image Processing, Vol. 9, Number 8, 2015, pp.615625.

[2] Moniruzzaman, Hawladar, Hossain, Md., "Wavelet Based Watermarking Approach for Hiding Patient Information in medical Image for Medical Image Authentication", $17^{\text {th }}$ International Conference on Computer and Information Technology (ICCIT), 22-23 December, 2014.

[3] Paulopoulas, G. S., Kaoutsouris, D., "Multiple Image Watermarking Scheme Applied to Medical Image Management", IEEE Trans. on Information Technology in Biomedicine, Vol. 10, Number 4, pp. 3241-3244, 2006.

[4] Dong, P., "Robust Digital Image Watermarking", PhD thesis, Electrical Engineering in the Graduate College of the Illinois Institute of Technology, May 2004.

[5] O. Jane, H.K. Ilk, E. Elbasi, "A Secure and Robust Watermarking Algorithm Based on the Combination of DWT, SVD and LU Decomposition with Arnold's Cat Map Approach", 8th International Conference on Electrical and Electronics Engineering (ELECO), 28-30 November, 2013.

[6] S. Haykin, "Adaptive Filter Theory", Prentice Hall, New Jersey, 4.th edt., 2002.

[7] Q. Li, C. Yuan, Y. Zhong, "Adaptive DWT-SVD Domain Image Watermarking Using Human Visual Model", The $9^{\text {th }}$ International on Advanced Communication Technology, 12-14 February, 2007.

[8] K. Pal, G. Ghosh, M. Bhattacharya, "Retrieval of hidden infected region using biomedical image watermarking for telediagnosis to ensure better treatment", $5^{\text {th }}$ International Conference on Computers and Devices for Communication (CODEC), 17-19 December, 2012.

[9] K. Pal, G. Ghosh, M. Bhattacharya, "Biomedical Image Watermarking for Content Protection Using Multiple Copies of Information and Bit Majority Algorithm in Wavelet Domain",

IEEE Student's Conference on Electrical, Electronics and Computer Science (SCEECS), 1-2 March, 2012.

[10] X. Xi'an, W. Yongsheng, "Study on Algorithm of Masking Digital Watermarking in Wavelet Transform Domain Based on Chaos Encryption and Arnold Transform", International Conference on Audio Language and Image Processing (ICALIP), 23-25 November, 2010.

\section{References}

